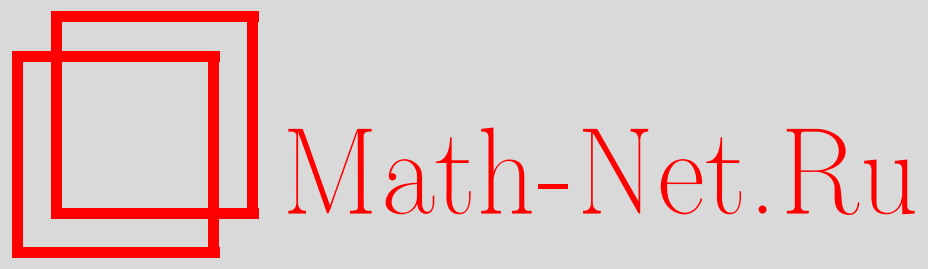

Ю. А. Неретин, Сферичность и умножение двойных классов смежности для бесконечномерных классических групп, Функи, анализ и его прил., 2011, том 45, выпуск 3, 79-96

DOI: https://doi.org/10.4213/faa3042

Использование Общероссийского математического портала MathNet.Ru подразумевает, что вы прочитали и согласны с пользовательским соглашением

http://www . mathnet.ru/rus/agreement

Параметры загрузки:

IP : 35.173 .219 .12

26 апреля 2023 г., 11:57:34

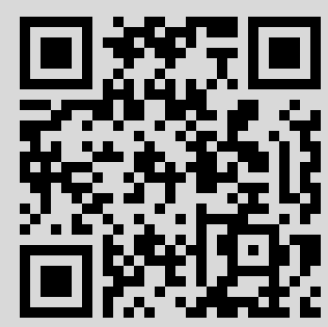




\title{
Сферичность и умножение двойных классов смежности для бесконечномерных классических групп*
}

\author{
(c) 2011. Ю. А. НЕРЕТин
}

\author{
Памяти В. И. Арнольда
}

\begin{abstract}
Мы строим сферические подгруппы в бесконечномерных классических группах $G$ (как правило, они не симметрические, и их аналоги в конечномерном случае сферическими не являются). Предъявляется структура полугруппы на пространствах двойных классов смежности $L \backslash G / L$ для различных подгрупп $L$ в $G$, причем эти полугруппы действуют в пространствах $L$-неподвижных векторов в унитарных представлениях групп $G$. Строятся также полугрупповые оболочки групп $G$, обобщающие конструкции операторных узлов.
\end{abstract}

\section{§1. Введение}

1.1. Определение сферичности. Пусть $G$ - группа, $K \subset G$ - подгруппа. Неприводимое унитарное представление $\rho$ группы $G$ в гильбертовом пространстве $H$ называется $K$-сферическим, если в $H$ есть единственный с точностью до пропорциональности $K$-неподвижный вектор $v$. Сферическая функиия сферического представления задается формулой

$$
\Phi(g)=\langle\rho(g) v, v\rangle .
$$

Пусть $G$ - полупростая группа Ли, а $K$ - ее компактная подгруппа. Пара $(G, K)$ называется сферической, если любое неприводимое унитарное представление группы $G$ содержит не более одного ненулевого $K$-неподвижного вектора. Говорят также, что $K-$ сферическая подгруппа группы $G$.

Согласно теореме Гельфанда [5], симметрические подгруппы ${ }^{1)}$ сферичны. В частности, сферичны максимальные компактные подгруппы конечномерных полупростых групп.

Кремер [12] рассклассифицировал сферические пары $(G, K)$ с простой группой $G$; классификация была распространена на полупростые группы в [15] и [2]. Все такие пары получаются из симметрических пар $(G, K)$ небольшим уменьшением подгруппы $K$ или небольшим увеличением $G$. O сферических функциях для несимметрических пар см. работу Кнопа [10].

1.2. Бесконечномерные классические группы. Мы рассматриваем гильбертовы пространства $l_{2}$ над $\mathbb{R}, \mathbb{C}$ и над кватернионами $\mathbb{H}$. Назовем матрицу $g$ с элементами из $\mathbb{R}, \mathbb{C}, \mathbb{H}$ финитной, если матрица $g-1$ имеет лишь конечное число ненулевых элементов. Мы определяем бесконечномерные классические

* Поддержано грантом FWF P22122.

1) Пусть $G$ - группа, а $\sigma-$ ее инволютивный автоморфизм, $\sigma^{2}=1$. Множество неподвижных точек автоморфизма $\sigma$ образует подгруппу, такие подгруппы называются симметрическими. Подробности и классификацию см. в [7]. 
группь $\mathrm{U}(\infty), \mathrm{O}(\infty), \mathrm{GL}(\infty, \mathbb{R}), \mathrm{Sp}(2 \infty, \mathbb{R}), \mathrm{U}(\infty, \infty), \ldots$ как группы финитных матриц, удовлетворяющих обычным соотношениям. Все такие группы $G(\infty)$ являются индуктивными пределами соответствующих конечномерных групп $G(n)$,

$$
\operatorname{Sp}(2 \infty, \mathbb{R})=\lim _{\longrightarrow} \operatorname{Sp}(2 n, \mathbb{R}), \quad \mathrm{U}(\infty, \infty)=\underline{\lim _{\longrightarrow}} \mathrm{U}\left(n_{1}, n_{2}\right), \quad \cdots
$$

Мы считаем, что $G(n)$ канонически вложены в $G(\infty)$ и действуют на первые базисные векторы. Если мы рассматриваем группы типа $\mathrm{O}(\infty, \infty)$ и т. п. или произведения групп, то $n$ в $G(n)$ понимается как $n=\left(n_{1}, \ldots\right)$.

1.3. Тяжелые группы. Ортогональная группа $\mathrm{O}(\infty)$, унитарная группа $\mathrm{U}(\infty)$ и кватернионно-унитарная группа $\mathrm{Sp}(\infty)$ играют ту же роль, что компактные группы в конечномерной теории. Мы будем называть их тлжслыми. Для тяжелой группы $K$ мы обозначим через $K^{\alpha}$ стабилизатор первых $\alpha$ базисных элементов в соответствующем $\ell_{2}$. Мы изображаем элементы из $K^{\alpha}$ как блочные $(\alpha+\infty) \times(\alpha+\infty)$-матрицы вида $\left(\begin{array}{ll}1 & 0 \\ 0 & h\end{array}\right)$, где $h \in K$.

Пусть $\rho$ - унитарное представление тяжелой группы $K$ в гильбертовом пространстве $H$. Обозначим через $H^{[\alpha]}$ подпространство $K^{\alpha}$-неподвижных векторов.

Теорема 1.1 [25]. Следующие условия равносильнь:

- $\rho$ непрерывно относительно слабой топологии на $K$;

- $\rho$ непрерывно относительно равномерной топологии на $K$;

- U $H^{[\alpha]}$ плотно в $H$;

- $\rho$ продолжается до представления полугруппы операторов в $\ell_{2}$ с нормой ${ }^{1)}$ $\leqslant 1$, снабженной слабой операторной топологией.

Если $\rho$ неприводимо, то эти условия равносильны такэе условию

- $H^{[\alpha]} \neq 0$ для какого нибудъ $\alpha$.

Такие представления мы будем называть непрерывными. Описание непрерывных представлений содержится в [25], все они получаются тензорными операциями из простейшего представления группы $K$ в $\ell_{2}$.

Конечные произведения тяжелых групп мы тоже будем называть тяљельми. Если $K=K_{1} \times \cdots \times K_{p}$, то мы полагаем $K^{\alpha}:=K_{1}^{\alpha_{1}} \times \cdots \times K_{p}^{\alpha_{p}}$, где $\alpha=\left(\alpha_{1}, \ldots, \alpha_{p}\right)$ - мультииндекс. Неприводимые представления тяжелой группы $K=K_{1} \times \cdots \times K_{p}$ являются тензорными произведениями представлений əpynn $K_{j}$.

1.4. Список Ольшанского. Следующий список состоит из пар $(G, K)=$ (классическая группа, симметрическая тяжелая подгруппа). Явный вид вложений $K(\infty) \rightarrow G(\infty)$ достаточно очевиден.

Пары некомпактного типа:

$$
\begin{aligned}
& (\mathrm{GL}(\infty, \mathbb{R}), \mathrm{O}(\infty)), \\
& (\mathrm{GL}(\infty, \mathbb{C}), \mathrm{U}(\infty)), \\
& (\mathrm{GL}(\infty, \mathbb{H}), \mathrm{Sp}(\infty)), \\
& (\mathrm{U}(\infty, \infty), \mathrm{U}(\infty) \times \mathrm{U}(\infty)), \\
& (\mathrm{Sp}(2 \infty, \mathbb{R}), \mathrm{U}(\infty)),
\end{aligned}
$$

Пары компактного типа:

$$
\begin{aligned}
& (\mathrm{U}(\infty), \mathrm{O}(\infty)), \\
& (\mathrm{U}(\infty) \times \mathrm{U}(\infty), \mathrm{U}(\infty)), \\
& (\mathrm{U}(2 \infty), \mathrm{Sp}(\infty)), \\
& (\mathrm{U}(2 \infty), \mathrm{U}(\infty) \times \mathrm{U}(\infty)), \\
& (\mathrm{Sp}(\infty), \mathrm{U}(\infty)),
\end{aligned}
$$

\footnotetext{
1) Такие операторы мы будем называть сжатиями.
} 

$(\operatorname{Sp}(2 \infty, \mathbb{C}), \operatorname{Sp}(\infty))$,
$(\operatorname{Sp}(\infty) \times \operatorname{Sp}(\infty), \operatorname{Sp}(\infty))$,
$(\operatorname{Sp}(\infty, \infty), \operatorname{Sp}(\infty) \times \operatorname{Sp}(\infty))$,
$(\operatorname{Sp}(2 \infty), \operatorname{Sp}(\infty) \times \operatorname{Sp}(\infty))$,
$(\mathrm{O}(\infty, \infty), \mathrm{O}(\infty) \times \mathrm{O}(\infty))$
$(\mathrm{O}(2 \infty), \mathrm{O}(\infty) \times \mathrm{O}(\infty))$,
$(\mathrm{O}(\infty, \mathbb{C}), \mathrm{O}(\infty))$
$(\mathrm{O}(\infty) \times \mathrm{O}(\infty), \mathrm{O}(\infty))$
$\left(\mathrm{SO}^{*}(2 \infty), \mathrm{U}(\infty)\right)$.
$(\mathrm{O}(2 \infty), \mathrm{U}(\infty))$.

Есть еще три серии симметрических пар «конечного ранга»:
$(\mathrm{U}(p, \infty), \mathrm{U}(p) \times \mathrm{U}(\infty))$,
$(\mathrm{U}(p+\infty), \mathrm{U}(p) \times \mathrm{U}(\infty))$,
$(\mathrm{O}(p, \infty), \mathrm{O}(p) \times \mathrm{O}(\infty))$,
$(\mathrm{O}(p+\infty), \mathrm{O}(p) \times \mathrm{O}(\infty))$,
$(\operatorname{Sp}(p, \infty), \operatorname{Sp}(p) \times \operatorname{Sp}(\infty))$,
$(\operatorname{Sp}(p+\infty), \operatorname{Sp}(p) \times \operatorname{Sp}(\infty))$.

В парах $(G, K)$ левого столбца группа $G$ пробегает все неприводимые бесконечномерные классические группь ${ }^{1)}$. В первой таблице в первом столбце подгруппа $K$ - максимальная тяжелая подгруппа в $G$. Максимальные тяжелая подгруппа в $\mathrm{U}(p, \infty)$ - это $\mathrm{U}(\infty)$.

Теорема сферичности. Все перечисленные выше пары $(G, K)$ являются сферическими.

Сферические представления расклассифицированы, см. [35], [26], [28], [27], [32], сферические функции задаются простыми явными формулами. Рассмотрим, например, $(\mathrm{GL}(\infty, \mathbb{R}), \mathrm{O}(\infty))$. Сферические представления определяются конечными наборами вещественных параметров $s_{1}, \ldots, s_{p}$, а также $a \in \mathbb{R}$ и $\sigma=0,1$. Сферические функции задаются формулой

$$
\Phi_{s, a, \sigma}(g)=|\operatorname{det} g|^{i a} \cdot \operatorname{sgn}(\operatorname{det} g)^{\sigma} \prod_{k} \prod_{l}\left(\frac{1+i s_{k}}{2} \lambda_{l}+\frac{1-i s_{k}}{2} \lambda_{l}^{-1}\right)^{-1 / 2},
$$

где через $\lambda_{j}$ обозначены сингулярные числа матрицы $g \in \mathrm{GL}(\infty, \mathbb{R})$, a sgn обозначает знак.

Замечание. Для пары $(\mathrm{U}(\infty) \times \mathrm{U}(\infty), \mathrm{U}(\infty))$ в настоящее время построен содержательный гармонический анализ ([31], [1]), он должен существовать и для других пар правого столбца, см. [18].

Класс сферических представлений не замкнут относительно естественных операций (тензорных произведений, ограничений на подгруппы), и его естественно расширить.

1.5. Допустимые представления. Фиксируем пару $(G, K)$ из списка. Назовем унитарное представление группы $G$ допустимым [28], если оно непрерывно на тяжелой подгруппе $K$. Мы будем употреблять в качестве синонима термин представление пары $(G, K)$. Разумеется, допустимость можно определять в терминах подходящих топологий на $G$ (см. обсуждение в [17]).

Лемма 1.2. Сферические представления допустимы.

Доказательство. Легко видеть, что подпространство $\bigcup_{\alpha} H^{[\alpha]}$ инвариантно относительно $G$. С другой стороны, оно непусто (сферический вектор есть). В силу неприводимости представления оно плотно.

1) Разумеется, группы $\mathrm{U}(\infty), \mathrm{O}(\infty), \mathrm{Sp}(\infty)$ - тоже классические, $p=0$. Неприводимая группа - это группа, не разлагающаяся в прямое произведение. 
Подчеркнем, что группы финитных матриц $\mathrm{GL}(\infty, \mathbb{R}), \mathrm{U}(\infty), \mathrm{O}(\infty, \mathbb{C})$ и т. п. не являются группами типа $I$ и теория их унитарных представлений в обычном смысле слова невозможна ${ }^{1)}$. Класс допустимых представлений обозрим, и содержательные работы по представлениям бесконечномерных классических групп в основном укладываются в эту схему.

Замечание. В списке присутствуют, например, пары $(\mathrm{O}(2 \infty), \mathrm{O}(\infty) \times \mathrm{O}(\infty))$, $(\mathrm{O}(2 \infty), \mathrm{U}(\infty))$. Группа $G$ в них одна и та же, однако условия непрерывности относительно $K$ различны (см. [28]). Например, неодномерное сферическое представление одной пары не является допустимым для другой.

Замечание. В списке есть пара $(\mathrm{U}(\infty) \times \mathrm{U}(\infty), \mathrm{U}(\infty))$. Ее представления не разлагаются в тензорные произведения $\rho_{1} \otimes \rho_{2}$ представлений группы $\mathrm{U}(\infty)$ (соответствующая конечномерная теорема, см. [6, 13.11.8], здесь «не работает»), о представлениях этой пары см. [34], [35], [28].

1.6. Умножение двойных классов смежности и шлейф. Пусть $\rho-$ допустимое представление пары $(G, K)$ в пространстве $H$. Пусть по-прежнему $H^{[\alpha]}$ - подпространства $K^{\alpha}$-неподвижных векторов. Обозначим через $P^{[\alpha]}$ оператор ортогонального проектирования на $H^{[\alpha]}$. Определим операторы

$$
\bar{\rho}_{\alpha, \beta}(g)=P^{[\beta]} \rho(g): H^{[\alpha]} \rightarrow H^{[\beta]} .
$$

Легко видеть, что для любых $h_{1} \in K^{\alpha}, h_{2} \in K^{\beta}$ выполнено равенство $\bar{\rho}_{\alpha, \beta}\left(h_{2} g h_{1}\right)$ $=\bar{\rho}_{\alpha, \beta}(g)$. Поэтому функция $\bar{\rho}_{\alpha, \beta}$ определена на двойных классах смежности $\mathfrak{g} \in K^{\beta} \backslash G / K^{\alpha}$.

Теорема мультипликативности. Существует естественное ассоииативное умножение $(\mathfrak{g}, \mathfrak{h}) \mapsto \mathfrak{g} \circ \mathfrak{h}$,

$$
K^{\gamma} \backslash G / K^{\beta} \times K^{\beta} \backslash G / K^{\alpha} \longrightarrow K^{\gamma} \backslash G / K^{\alpha},
$$

определенное для всех $\alpha, \beta, \gamma \in \mathbb{Z}_{+}$. Более того, для любого унитарного допустимого представления $\rho$ пары $(G, K)$

$$
\bar{\rho}_{\beta, \gamma}(\mathfrak{g}) \bar{\rho}_{\alpha, \beta}(\mathfrak{h})=\bar{\rho}_{\alpha, \gamma}(\mathfrak{g} \circ \mathfrak{h}) .
$$

Мы получили категорию, которую назовем шлейбом $\mathfrak{T}(G, K)$ пары $(G, K)$. Ее объекты - индексы (мультииндексы) $\alpha \geqslant 0$, а морфизмы - двойные классы смежности. Формула (1.1) означает, что мы получили представление категории (см. $[17$, II.5]), т. е. функтор из $\mathfrak{T}(G, K)$ в категорию гильбертовых пространств и ограниченных линейных операторов.

Отображение $g \mapsto g^{-1}$ индуцирует отображение $K^{\alpha} \backslash G / K^{\beta} \rightarrow K^{\beta} \backslash G / K^{\alpha}$, мы обозначим его через $\mathfrak{g} \mapsto \mathfrak{g}^{*}$. Оно удовлетворяет условию

$$
(\mathfrak{g} \circ \mathfrak{h})^{*}=\mathfrak{h}^{*} \circ \mathfrak{g}^{*} .
$$

Представление $\bar{\rho}$ является *-представлением в следующем смысле: $\rho\left(\mathfrak{g}^{*}\right)=$ $\rho(\mathfrak{g})^{*}$.

1) Определение групп типа $I$, см., например, в [6]. Для групп не типа $I$ множество унитарных представлений необозримо, разложение приводимого представления не единственно и множество всех таких разложений, в свою очередь, необозримо. 
Свойство аппроксимации. Рассмотрим *-представление $\tau$ категории $\mathfrak{T}(G, K)$. Пусть выполнены условия $\|\tau(\mathfrak{g})\| \leqslant 1, \tau(1)=1$. Тогда представление $\tau$ имеет вид $\bar{\rho}$ для некоторого однозначно определенного представления $\rho$ napıь $(G, K)$.

1.7. Сравнение с конечномерным случаем. Пусть $G$ - группа Ли, а $L$ - ее компактная подгруппа, не обязательно максимальная. Обозначим через $\mathcal{M}(L \backslash G / L)$ пространство конечных мер, инвариантных относительно левых и правых сдвигов на элементы из $L$. Очевидно, что $\mathcal{M}(L \backslash G / L)$ - алгебра относительно свертки. Например, если $G$ - это $p$-адическая $\mathrm{GL}(n)$, a $L$ - ее ивахорическая подгруппа, мы получаем алгебру Гекке (см. [14]); если $G$ - вещественная простая группа Ли ранга 1 , а $L$ - максимальная компактная подгруппа, то получается гипергруппа операторов обобщенного сдвига [11].

Пусть $\rho$ - унитарное представление группы $G$ в пространстве $H$. Обозначим через $H^{L}$ пространство $L$-неподвижных векторов. Для $\mu \in \mathcal{M}(L \backslash G / L)$ оператор $\rho(\mu)$ имеет блочную форму $\left(\begin{array}{cc}A(\mu) & 0 \\ 0 & 0\end{array}\right)$ относительно разложения $H=$ $H^{L} \oplus\left(H^{L}\right)^{\perp}$. То есть мы получили функтор из унитарных представлений группы $G$ в представления сверточной алгебры $\mathcal{M}(L \backslash G / L)$.

Оказывается, что для бесконечномерных групп операция свертки двойных классов смежности может вырождаться в умножение. Первый пример, видимо, был обнаружен Исмагиловым в [8], [9], далее примеры появлялись в [23], [25], [28], [16]; многочисленные примеры обсуждаются в [17].

1.8. Явный вид произведения. См. [28]. Рассмотрим для определенности пару $(G, K)=(\mathrm{GL}(\infty, \mathbb{R}), \mathrm{O}(\infty))$ и двойные классы смежности $K^{\beta} \backslash G / K^{\alpha}$. Иными словами, мы рассматриваем матрицы $\mathfrak{g}=\left(\begin{array}{ll}A & B \\ C & D\end{array}\right)$ размера $(\beta+\infty) \times(\alpha+\infty)$ с точностью до эквивалентности

$$
\left(\begin{array}{ll}
A & B \\
C & D
\end{array}\right) \sim\left(\begin{array}{cc}
1_{\beta} & 0 \\
0 & U
\end{array}\right)\left(\begin{array}{cc}
A & B \\
C & D
\end{array}\right)\left(\begin{array}{cc}
1_{\alpha} & 0 \\
0 & V
\end{array}\right)
$$

где $U, V$ - ортогональные матрицы, а $1_{\alpha}$ обозначает единичную матрицу размера $\alpha$. Умножение в категории $\mathfrak{T}$ задается формулой

$$
\left(\begin{array}{ll}
A & B \\
C & D
\end{array}\right) \circ\left(\begin{array}{cc}
P & Q \\
R & T
\end{array}\right):=\left(\begin{array}{ccc}
A & B & 0 \\
C & D & 0 \\
0 & 0 & 1
\end{array}\right)\left(\begin{array}{ccc}
P & 0 & Q \\
0 & 1 & 0 \\
R & 0 & T
\end{array}\right)=\left(\begin{array}{ccc}
A P & B & A Q \\
C P & D & C Q \\
R & 0 & T
\end{array}\right) .
$$

Отметим, что похожая (но не буквально та же) алгебраическая структура известна как умножение операторных узлов, см., например, [3].

1.9. Характеристические функции. Оказывается (см. [17, IX.4]), что о-умножение можно преобразовать в более привычную операцию ${ }^{1)}$ следующим

1)Это аналог характеристической функции Лившица, известной в спектральной теории несамосопряженных операторов, см. [13], [33]. Сама функция Лифшица участвует в теории представлений пары $(\mathrm{U}(1+\infty) \times \mathrm{U}(\infty), \mathrm{U}(\infty))$, см. [30]. 
способом. Фиксируем $\lambda \in \mathbb{C} \cup \infty$. Напишем уравнение

$$
\left(\begin{array}{c}
p_{+} \\
\lambda x \\
p_{-} \\
x
\end{array}\right)=\left(\begin{array}{cccc}
A & B & & 0 \\
C & D & & \\
& 0 & & \left(\begin{array}{cc}
A & B \\
C & D
\end{array}\right)^{t-1}
\end{array}\right)\left(\begin{array}{c}
q_{+} \\
y \\
q_{-} \\
\lambda y
\end{array}\right) .
$$

Рассмотрим все векторы $\left(p_{+}, p_{-}\right) \oplus\left(q_{+}, q_{-}\right) \in \mathbb{C}^{2 \beta} \oplus \mathbb{C}^{2 \alpha}$, для которых это уравнение имеет решение как уравнение на $x, y$. Мы получаем подпространство $\chi(\lambda)$ половинной размерности в $\mathbb{C}^{2 \beta} \oplus \mathbb{C}^{2 \alpha}$, зависящее от $\lambda$, которое мы рассматриваем как отношение (многозначное отображение) из $\mathbb{C}^{2 \alpha}$ в $\mathbb{C}^{2 \beta}$.

На этом языке о-умножение соответствует произведению характеристических функций, т. е. поточечному произведению отношений.

Отметим, что $\chi(\lambda)$ является рациональным отображением сферы Римана в лагранжев грассманиан в $\mathbb{C}^{2 \alpha} \oplus \mathbb{C}^{2 \beta}$. Функция $\chi(\lambda)$ является также $J$-сжимающей в смысле Потапова [33].

1.10. Самоподобие. См. [29]. Пусть $(G, K)=(\mathrm{GL}(\infty, \mathbb{R}), \mathrm{O}(\infty))$. Обозначим через $G^{\alpha} \subset G$ подгруппу в $G$, состоящую из всех матриц вида $\left(\begin{array}{cc}1_{\alpha} & 0 \\ 0 & g\end{array}\right)$. Тогда пара $\left(G^{\alpha}, K^{\alpha}\right)$ изоморфна $(G, K)$. Похожие подгруппы определены и во всех остальных случаях, только определять их надо чуть аккуратней: мы берем базисные векторы, оставляемые на месте подгруппой $K^{\alpha}$, и их стабилизатор в $G$.

Фиксируем неприводимое унитарное представление $\rho$ пары $(G, K)$. Его ограничение на достаточно малую подгруппу $\left(G^{\alpha}, K^{\alpha}\right) \simeq(G, K)$ содержит сферическое подпредставление. Все сферические подпредставления пары $(G, K)$, полученные таким образом, эквивалентны (для всех $\alpha)$.

1.11. Мантия. Пусть $(G, K)=(\mathrm{GL}(\infty, \mathbb{R}), \mathrm{O}(\infty))$. Разобьем $\mathbb{N}$ на два счетных подмножества, $\mathbb{N}=\Xi \cup \Omega$ (скажем, на нечетные и четные числа). Обозначим через $G^{\Xi} \subset G$ (соответственно $\left.G(\Xi)\right)$ стабилизатор базисных векторов, занумерованных элементами из $\Xi\left(\right.$ соответственно $\Omega$ ). Иными словами, $G^{\Xi}$ и $G(\Xi)$ состоят соответственно из матриц вида $\left(\begin{array}{ll}1 & 0 \\ 0 & g\end{array}\right)$ и $\left(\begin{array}{ll}g & 0 \\ 0 & 1\end{array}\right)$, где блоки отвечают разложению $\ell_{2}(\mathbb{N})=\ell_{2}(\Xi) \oplus \ell_{2}(\Omega)$. Очевидно, что $(G(\Xi), K(\Xi))$ изоморфна $(G, K)$. Мы фиксируем изоморфизм

$$
i_{\tau}:(G, K) \rightarrow(G(\Xi), K(\Xi)),
$$

индуцированный какой-нибудь биекцией $\tau: \mathbb{N} \rightarrow \Xi$.

Пусть $\rho$ - унитарное представление пары $(G, K)$ в пространстве $H$. Обозначим через $H^{[\Xi]}$ подпространство $K^{\Xi}$-неподвижных векторов. Через $P^{[\Xi]}$ мы обозначим проектор на $H^{[\Xi]}$.

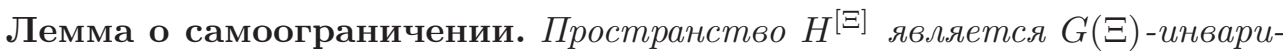
антным. Представление $\rho$ о $i_{\tau}$ паръ $(G, K)$ в $H^{[\Xi]}$ эквивалентно $\rho$.

$\mathrm{C}$ другой стороны, по элементу $g \in G$ мы строим оператор $\bar{\rho}_{\Xi, \Xi}(g): H^{[\Xi]} \rightarrow$ $H^{[\Xi]}$ как $\bar{\rho}_{\Xi, \Xi}(g)=P^{[\Xi]} \rho(g)$. Как и выше, $\bar{\rho}$ есть функция на двойных классах смежности. 
Вариант теоремы мультипликативности. Множество двойных классов смежности $\Gamma_{\infty}=K^{\Xi} \backslash G / K^{\Xi}$ обладает естественной структурой полугруппь (мантия пары $(G, K))$, отображсение $\bar{\rho}_{\Xi, \Xi}$ есть представление полугруппы $\Gamma_{\infty}$.

Умножение задается той же формулой (1.3), только теперь все блоки матрицы бесконечны.

Теперь заметим, что группа $G(\Xi) \simeq G$ вкладывается в $K^{\Xi} \backslash G / K^{\Xi}$ изоморфно, и, таким образом, мы можем считать, что представление полугруппы $K^{\Xi} \backslash G / K^{\Xi}$ продолжает представление $\rho$ группы $G(\Xi) \simeq G$.

В итоге мы получаем следующий результат:

Теорема о мантии. Любое унитарное представление пары $(G, K)$ канонически продолжсается до представления полугруппь $\Gamma_{\infty}$.

1.12. Сферический характер. Теперь заметим, что $Z:=K^{\Xi} \backslash G^{\Xi} / K^{\Xi} \subset$ $K^{\Xi} \backslash G / K^{\Xi}$ является центральной подполугруппой в мантии. В любом неприводимом представлении $\rho$ пары $(G, K)$ она действует скалярными операторами. Поэтому определен мультипликативный характер $\Phi_{\rho}: Z \rightarrow \mathbb{C}^{\times}$.

Если $\rho$ сферично, то $\Phi_{\rho}$ совпадает со сферической функцией представления $\rho$, в общем случае он совпадает со сферическими функциями из разд. 1.10.

1.13. Нестандартные пары. До сих пор мы говорили о парах $(G, K)$ из списка Ольшанского. Положим теперь $G=\mathrm{GL}(\infty, \mathbb{R}) \times \cdots \times \mathrm{GL}(\infty, \mathbb{R})$, и пусть $K \subset G$ - группа $\mathrm{O}(\infty)$, вложенная в $G$ по диагонали.

(a) Пара $(G, K)$ сферична. Отметим, что у этого утверждения нет конечномерных аналогов. Для этой пары верна и теорема мультипликативности.

(b) Нессонов ([21], [22]) получил описание всех сферических представлений этой пары.

(c) В [20] получена конструкция характеристических функций, пригодная в данном случае.

Встает вопрос: в какой общности верны приведенные выше (разд. 1.4-1.12). утверждения?

1.14. Цели работы. Мы вводим класс $(G, L)$-nар, где $G$ - классическая группа, $L$ - тяжелая подгруппа, для которых остаются в силе

- теорема мультипликативности,

- свойство аппроксимации,

- конструкция мантии,

- существование сферических характеров.

Кроме того, для них «работает» конструкция характеристических функций из $[20]$.

Мы вводим подкласс чистых $(G, L)$-nар, для которых дополнительно выполнены

- теорема о сферичности,

- свойство самоподобия.

Определение $(G, L)$-пар содержится в $\S 2$, доказательства - в $\S 3$ (повторно мы теорем не формулируем), §4 содержит гипотезы и дополнительные комментарии. 


\section{§2. Определения}

2.1. Правильные вложения тяжелых групп. Пусть сначала тяжелые группы $L, K$ неприводимы, т. е. имеют вид $\mathrm{U}(\infty), \mathrm{O}(\infty), \operatorname{Sp}(\infty)$. Имеются очевидные гомоморфизмы

$$
\begin{array}{lll}
\mathrm{O}(\infty) \rightarrow \mathrm{U}(\infty), & \mathrm{O}(\infty) \rightarrow \mathrm{Sp}(\infty), & \mathrm{U}(\infty) \rightarrow \mathrm{Sp}(\infty), \\
\mathrm{U}(\infty) \rightarrow \mathrm{O}(2 \infty), & \mathrm{Sp}(\infty) \rightarrow \mathrm{O}(4 \infty), & \mathrm{Sp}(\infty) \rightarrow \mathrm{U}(2 \infty) .
\end{array}
$$

Кроме того, поэлементное комплексное сопряжение определяет изоморфизм группы $\mathrm{U}(\infty)$ на себя. Такие гомоморфизмы, а также тождественные гомоморфизмы $g \mapsto g$, мы будем называть тривиальными.

Пусть теперь $K$ неприводима, а $L=L_{1} \times \cdots \times L_{p}$. Гомоморфизм $L \rightarrow K$ мы назовем правилъным, если он имеет вид

$$
\left(g_{1}, \ldots, g_{p}\right) \mapsto\left(\begin{array}{cccc}
1_{a} & 0 & \ldots & 0 \\
0 & \tau_{1}\left(g_{m_{1}}\right) & \ldots & 0 \\
\vdots & \vdots & \ddots & \vdots \\
0 & 0 & \ldots & \tau_{N}\left(g_{m_{N}}\right)
\end{array}\right), \quad g_{k} \in L_{k},
$$

где $\tau_{j}$ - тривиальные гомоморфизмы. Единичная матрица $1_{a}$ может иметь любой размер $0,1,2, \ldots, \infty$. Число $N$ может быть любым в пределах $0<N<\infty$. Гомоморфизм не обязательно является точным.

Пусть теперь $L=L_{1} \times \cdots \times L_{p}, K=K_{1} \times \cdots \times K_{q}$. Тогда правильный гомомор$\oint и з м$ - это произведение правильных гомоморфизмов $L \rightarrow K_{1}, \ldots, L \rightarrow K_{q}$.

2.2. $(\boldsymbol{G}, \boldsymbol{L})$-пары. Назовем классической группой $G=G_{1} \times \cdots \times G_{r}$ конечное произведение неприводимых классических групп (список приведен выше). Пусть $K=K_{1} \times \cdots \times K_{q}$ - максимальная тяжелая подгруппа в $G$. Пусть $L-$ правильно вложенная тяжелая подгруппа в $K$. Полученный объект $(G, L)$ мы будем называть $(G, L)$-парой.

Пару $(G, L)$ мы будем называть чистой, если централизатор подгруппы $L$ в $G$ тривиален ${ }^{1)}$. Равносильное условие: в матрицах (2.1) отсутствует единичный блок $1_{a}$, а среди множителей $G_{j}$ отсутствуют группы $\mathrm{U}(p, \infty), \mathrm{O}(p, \infty), \operatorname{Sp}(p, \infty)$ c $p>0^{2}$.

Назовем пару конечной, если блоки $1_{a}$ в формуле (2.1) для всех множителей $K_{i}$ имеют конечные размеры. Конечные пары появятся лишь в гипотезах в последнем параграфе.

2.3. Определение умножения двойных классов смежности. Пусть $L$ неприводима. Фиксируем $\alpha \geqslant 0$ и рассмотрим последовательность $\Theta_{m}^{[\alpha]} \in L$,

1) Например, пара $(G, L)=(\mathrm{GL}(3 \infty, \mathbb{R}), \mathrm{O}(\infty) \times \mathrm{O}(\infty) \times \mathrm{O}(\infty))$ чистая. Централизатор подгруппы $L$ в группе всех ограниченных операторов равен $\left(\mathbb{R}^{\times}\right)^{3}$. Но он не содержится в группе финитных матриц $G=\mathrm{GL}(3 \infty, \mathbb{R})$. Пара $(\mathrm{O}(1+3 \infty, \mathbb{R}), \mathrm{O}(\infty) \times \mathrm{O}(\infty) \times \mathrm{O}(\infty))$ не чистая, централизатор в $\mathrm{O}(1+3 \infty, \mathbb{R})$ изоморфен $\mathbb{Z}_{2}$.

2) Данную оговорку приходится произнести, потому что для этих групп максимальная тяжелая подгруппа не является точным аналогом максимальной компактной. 
заданную формулой

$$
\rho\left(\Theta_{m}^{[\alpha]}\right)=\left(\begin{array}{cccc}
1_{\alpha} & 0 & 0 & 0 \\
0 & 0 & 1_{m} & 0 \\
0 & 1_{m} & 0 & 0 \\
0 & 0 & 0 & 1_{\infty}
\end{array}\right)
$$

Если $L$ приводима, $L=L_{1} \times \cdots \times L_{p}$, то мы фиксируем мультииндекс $\alpha$ и рассматриваем направленность, индексированную мультииндексом $m$,

$$
\Theta_{m}^{[\alpha]}=\left(\Theta_{m_{1}}^{\left[\alpha_{1}\right]}, \ldots, \Theta_{m_{p}}^{\left[\alpha_{p}\right]}\right)
$$

Пусть

$$
\mathfrak{g} \in K^{[\gamma]} \backslash G / K^{[\beta]}, \quad \mathfrak{h} \in K^{[\beta]} \backslash G / K^{[\alpha]} .
$$

Выберем представителей $g \in \mathfrak{g}, h \in \mathfrak{h}$. Рассмотрим последовательность

$$
z_{m}=g \Theta_{m}^{[\beta]} h \in G \text {. }
$$

Предложение 2.1. (а) Двойной класс смежннсти $\mathfrak{z} m=K^{[\gamma]} z_{m} K^{[\alpha]}$ стабилизируется, начиная с некоторого места.

(b) Результат $g \circ h=\lim \mathfrak{z}$ не зависит от выбора представителей $g \in \mathfrak{g}$, $h \in \mathfrak{h}$.

(с) Полученная операция на двойных классах смежсности ассоииативна, m. $е$. для любых $\alpha, \beta, \gamma, \delta$ и для любъх

$$
\mathfrak{f} \in K^{\delta} \backslash G / K^{\gamma}, \quad \mathfrak{g} \in K^{\gamma} \backslash G / K^{\beta}, \quad \mathfrak{h} \in K^{\beta} \backslash G / K^{\alpha}
$$

въполнено равенство $(\mathfrak{f} \circ \mathfrak{g}) \circ \mathfrak{h}=\mathfrak{f} \circ(\mathfrak{g} \circ \mathfrak{h})$.

В итоге мы получаем категорию, которую мы по-прежнему обозначаем через $\mathfrak{T}(G, L)$ и называем шлейфом пары $(G, L)$.

Вместо формального доказательства для общего случая мы приведем примеры, показывающие, почему так получается.

2.4. Пример. Рассмотрим пару $(\mathrm{GL}(\infty, \mathbb{R}), \mathrm{O}(\infty))$. Для упрощения обозначений положим $\gamma=\beta=\alpha$. Возьмем $g, h \in G$. Запишем $g, h$ как блочные матрицы порядка $\alpha+N+\infty$. Пусть $N$ достаточно велико. Тогда $g, h$ имеют вид

$$
g=\left(\begin{array}{ccc}
a & b & 0 \\
c & d & 0 \\
0 & 0 & 1_{\infty}
\end{array}\right), \quad h=\left(\begin{array}{ccc}
p & q & 0 \\
r & t & 0 \\
0 & 0 & 1_{\infty}
\end{array}\right) .
$$

Произведение $g \Theta_{N+k}^{[\alpha]} h$ равно

$$
\left(\begin{array}{cccccc}
a p & a q & 0 & b & 0 & 0 \\
c p & c q & 0 & d & 0 & 0 \\
0 & 0 & 0 & 0 & 1_{k} & 0 \\
r & t & 0 & 0 & 0 & 0 \\
0 & 0 & 1_{k} & 0 & 0 & 0 \\
0 & 0 & 0 & 0 & 0 & 1_{\infty}
\end{array}\right)
$$

Так как мы рассматриваем двойные классы смежности, мы можем переставлять местами строчки (столбцы) этой матрицы с номерами $>\alpha$. Таким образом мы 
можем привести матрицу к одной (любой) из двух удобных форм

$$
S_{1}=\left(\begin{array}{cccc}
a p & a q & b & 0 \\
c p & c q & d & 0 \\
r & t & 0 & 0 \\
0 & 0 & 0 & 1_{2 k+\infty}
\end{array}\right), \quad S_{2}=\left(\begin{array}{cccc}
a p & b & a q & 0 \\
c p & d & c q & 0 \\
r & 0 & t & 0 \\
0 & 0 & 0 & 1_{2 k+\infty}
\end{array}\right) .
$$

Мы видим, что результат не зависит от $k$. Теперь надо проверить, что он не зависит от выбора представителей, т.е. что $g u, v h$ с $u, v \in K_{\alpha}$ дают тот же самый двойной класс смежности. Без ограничения общности мы можем считать, что $u, v \in \mathrm{O}(N)$ (иначе мы с самого начала возьмем большее $N$ ). Мы заменяем $g, h$ из (2.3) на

$$
g=\left(\begin{array}{ccc}
a & b u & 0 \\
c & d u & 0 \\
0 & 0 & 1
\end{array}\right), \quad h=\left(\begin{array}{ccc}
p & q & 0 \\
v r & v t & 0 \\
0 & 0 & 1
\end{array}\right)
$$

с ортогональными матрицами $u, v$. Тогда новая матрица $S_{2}$ равна

$$
\left(\begin{array}{cccc}
a p & b u & a q & 0 \\
c p & d u & c q & 0 \\
v r & 0 & v t & 0 \\
0 & 0 & 0 & 1
\end{array}\right)=\left(\begin{array}{cccc}
1 & & & \\
& 1 & & \\
& & v & \\
& & & 1
\end{array}\right)\left(\begin{array}{cccc}
a p & b & a q & 0 \\
c p & d & c q & 0 \\
r & 0 & t & 0 \\
0 & 0 & 0 & 1
\end{array}\right)\left(\begin{array}{cccc}
1 & & & \\
& u & & \\
& & 1 & \\
& & & 1
\end{array}\right)
$$

Ассоциативность ясна из формулы (1.3).

2.5. Два примера. (а) Рассмотрим пару, состоящую из группы $G=$ $\mathrm{GL}(\infty, \mathbb{R}) \times \cdots \times \mathrm{GL}(\infty, \mathbb{R})$ и ее диагональной подгруппы $L=\mathrm{O}(\infty)$. Мы имеем конечный набор матриц $g_{j}=\left(\begin{array}{ll}a_{j} & b_{j} \\ c_{j} & d_{j}\end{array}\right)$ с точностью до эквивалентности

$$
\begin{aligned}
\left\{\left(\begin{array}{ll}
a_{1} & b_{1} \\
c_{1} & d_{1}
\end{array}\right), \ldots,\left(\begin{array}{ll}
a_{q} & b_{q} \\
c_{q} & d_{q}
\end{array}\right)\right\} & \\
& \sim\left\{\left(\begin{array}{cc}
1_{\beta} & 0 \\
0 & u
\end{array}\right)\left(\begin{array}{ll}
a_{1} & b_{1} \\
c_{1} & d_{1}
\end{array}\right)\left(\begin{array}{cc}
1_{\alpha} & 0 \\
0 & v
\end{array}\right), \ldots,\left(\begin{array}{cc}
1_{\beta} & 0 \\
0 & u
\end{array}\right)\left(\begin{array}{cc}
a_{q} & b_{q} \\
c_{q} & d_{q}
\end{array}\right)\left(\begin{array}{cc}
1_{\alpha} & 0 \\
0 & v
\end{array}\right)\right\} .
\end{aligned}
$$

Тогда о-умножение определяется покомпонентно формулой (1.3).

(b) Пусть $G=\mathrm{U}(2 \infty), K=\mathrm{O}(\infty) \times \mathrm{O}(\infty)$. Теперь мы имеем унитарные матрицы $g$, определенные с точностью до эквивалентности

$$
\left(\begin{array}{llll}
a_{11} & b_{11} & a_{12} & b_{12} \\
c_{11} & d_{11} & c_{12} & d_{12} \\
a_{21} & b_{21} & a_{22} & b_{22} \\
c_{21} & d_{21} & c_{22} & d_{22}
\end{array}\right) \sim\left(\begin{array}{lllll}
1 & & & \\
& u_{1} & & \\
& & 1 & \\
& & u_{2}
\end{array}\right)\left(\begin{array}{llll}
a_{11} & b_{11} & a_{12} & b_{12} \\
c_{11} & d_{11} & c_{12} & d_{12} \\
a_{21} & b_{21} & a_{22} & b_{22} \\
c_{21} & d_{21} & c_{22} & d_{22}
\end{array}\right)\left(\begin{array}{llll}
1 & & \\
& v_{1} & & \\
& & 1 & \\
& & v_{2}
\end{array}\right),
$$

где $u_{1}, u_{2}, v_{1}, v_{2} \in \mathrm{O}(\infty)$. Произведение задается формулой

$$
g \circ g^{\prime}=\left(\begin{array}{cccccc}
a_{11} & b_{11} & 0 & a_{12} & b_{12} & 0 \\
c_{11} & d_{11} & 0 & c_{12} & d_{12} & 0 \\
0 & 0 & 1 & 0 & 0 & 0 \\
a_{21} & b_{21} & 0 & a_{22} & b_{22} & 0 \\
c_{21} & d_{21} & 0 & c_{22} & d_{22} & 0 \\
0 & 0 & 0 & 0 & 0 & 1
\end{array}\right)\left(\begin{array}{cccccc}
a_{11}^{\prime} & 0 & b_{11}^{\prime} & a_{12}^{\prime} & 0 & b_{12}^{\prime} \\
0 & 1 & 0 & 0 & 0 & 0 \\
c_{11}^{\prime} & 0 & d_{11}^{\prime} & c_{12}^{\prime} & 0 & d_{12}^{\prime} \\
a_{21}^{\prime} & 0 & b_{21}^{\prime} & a_{22}^{\prime} & 0 & b_{22}^{\prime} \\
0 & 0 & 0 & 0 & 1 & 0 \\
c_{21}^{\prime} & 0 & d_{21}^{\prime} & c_{22}^{\prime} & 0 & d_{22}^{\prime}
\end{array}\right) .
$$


Двойные классы смежности берутся относительно группы матриц вида

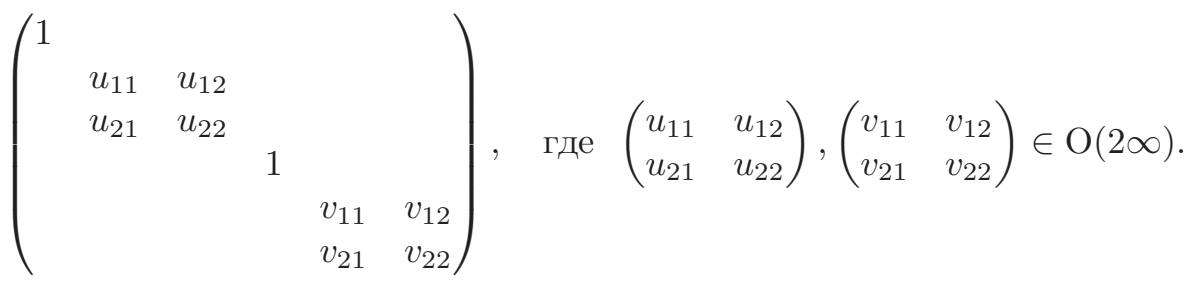

\section{§3. Доказательства}

3.1. Мультипликативность (аналог разд. 1.6).

Лемма 3.1. Для любого унитарного представления $\pi$ тяжелой группь $L$ последовательность $\pi\left(\Theta_{m}^{[\alpha]}\right)$ (см. (2.2)) слабо сходится $к$ проектору $P^{[\alpha]}$ на $H^{[\alpha]}$.

См. [17, теорема VIII.1.4]. Там априорное доказательство дано в случае симметрической группы $S(\infty)$, доказательство для групп $\mathrm{O}(\infty), \mathrm{U}(\infty), \operatorname{Sp}(\infty)$ такое же. С другой стороны, это утверждение легко выводится из явной классификации представлений [25] тяжелых групп, см. также [17].

Докажем теорему мультипликативности для произвольной пары $(G, L)$. Пусть $\mathfrak{g}, \mathfrak{h}, g, h$ - те же, что в разд. 2.3. Тогда

$$
\bar{\rho}(\mathfrak{g} \circ \mathfrak{h})=P^{[\gamma]} \rho\left(g \Theta_{m}^{[\alpha]} h\right): H^{[\alpha]} \rightarrow H^{[\gamma]}
$$

для достаточно больших $m$. С другой стороны,

$$
\begin{aligned}
\lim _{m \rightarrow \infty} P^{[\gamma]} \rho\left(g \Theta_{m}^{[\alpha]} h\right) & =\lim _{m \rightarrow \infty} P^{[\gamma]} \rho(g) \rho\left(\Theta_{m}^{[\alpha]}\right) \rho(h) \\
& =P^{[\gamma]} \rho(g)\left(\lim _{m \rightarrow \infty} \rho\left(\Theta_{m}^{[\alpha]}\right)\right) \rho(h)=P^{[\gamma]} \rho(g) P^{[\beta]} \rho(h)=\bar{\rho}(\mathfrak{g}) \bar{\rho}(\mathfrak{h}) .
\end{aligned}
$$

Итак, по каждому унитарному представлению пары $(G, L)$ мы построили представление категории $\mathfrak{T}(G, L)$. Теперь предъявим обратную конструкцию.

3.2. Свойство аппроксимации. Пусть $\alpha<\beta\left(\right.$ соответственно $\left.L^{\alpha} \supset L^{\beta}\right)$. Обозначим через $\lambda_{\alpha, \beta}$ двойной класс смежности $L^{\beta} \cdot 1 \cdot L^{\alpha}$. Положим $\mu_{\beta, \alpha}:=$ $\lambda_{\alpha, \beta}^{*}$. Тогда выполнены равенства

$$
\mu_{\beta, \alpha} \circ \lambda_{\alpha, \beta}=1^{[\alpha]}, \quad \lambda_{\beta, \gamma} \lambda_{\alpha, \beta}=\lambda_{\alpha, \gamma},
$$

где $1^{[\alpha]}$ обозначает единицу $L^{\alpha} \cdot 1 \cdot L^{\alpha}$ полугруппы $L^{\alpha} \backslash G / L^{\alpha}$. Мы получаем структуру упорядоченной категории в смысле [17, III.4].

Отметим, что $\psi_{\alpha, \beta}:=\lambda_{\alpha, \beta} \circ \mu_{\beta, \alpha}$ как подмножество в $G$ совпадает с $L^{\alpha}$. Но это нетривиальный идемпотент в $L^{\beta} \backslash G / L^{\beta}$ :

$$
\psi_{\alpha, \beta}^{2}=\psi_{\alpha, \beta}, \quad \psi^{*}=\psi_{\alpha, \beta} .
$$

Рассмотрим *-представление $R$ шлейфа $\mathfrak{T}(G, L)$ сжимающими операторами, и пусть единицы полугрупп эндоморфизмов переходят в единичные операторы. Обозначим через $H^{[\alpha]}$ гильбертово пространство, соответствующее (мульти)индексу $\alpha$. В силу (3.1) оператор $R\left(\lambda_{\alpha, \beta}\right)$ является оператором изометрического вложения $H^{[\alpha]} \rightarrow H^{[\beta]}$. Проектор на образ этого оператора - это 
$\psi_{\alpha, \beta}$. Теперь мы имеем цепочку изометричных вложений (для определенности $\alpha=\left(\alpha_{1}, \alpha_{2}\right)-$ бииндекс):

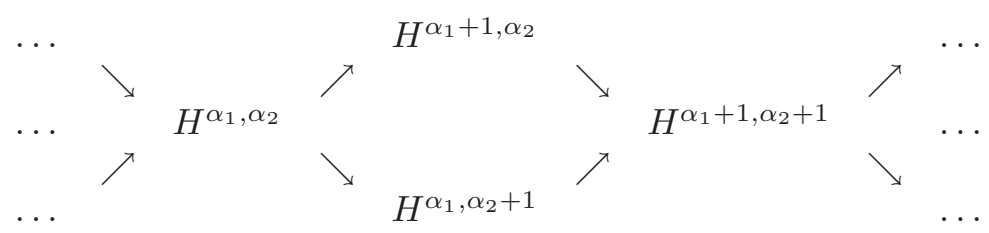

Предельное гильбертово пространство обозначим через $H$.

Группа $G$ является объединением конечномерных групп $G(\alpha)$. Заметим, что группа $G(\alpha)$ изоморфно вкладывается в полугруппу $L^{\alpha} \backslash G / L^{\alpha}$. Поэтому мы имеем представление группы $G(\alpha)$ в пространстве $H^{[\alpha]}$. Операторы представления являются сжимающими вместе со своими обратными. Следовательно, они унитарны.

Поэтому группа $G$ действует в $H$. Отслеживание дальнейших деталей мы опускаем.

\section{2. Сферичность.}

Лемма 3.2. Для чистых $(G, L)$-пар полугруппа $L \backslash G / L$ коммутативна.

Дело в том, что операция умножения в этом случае сводится к «приставлению» одной матрицы к другой. Например, формула (2.5) в этом случае сводится к такой:

$$
g \circ g^{\prime}=\left(\begin{array}{ll}
d_{11} & d_{12} \\
d_{21} & d_{22}
\end{array}\right) \circ\left(\begin{array}{ll}
d_{11}^{\prime} & d_{12}^{\prime} \\
d_{21}^{\prime} & d_{22}^{\prime}
\end{array}\right)=\left(\begin{array}{cccc}
d_{11} & 0 & d_{12} & 0 \\
0 & d_{11}^{\prime} & 0 & d_{12}^{\prime} \\
d_{21} & 0 & d_{22} & 0 \\
0 & d_{21}^{\prime} & 0 & d_{22}^{\prime}
\end{array}\right) \text {. }
$$

Чтобы получить $g^{\prime} \circ g$, мы сопрягаем правую часть матрицей

$$
\left(\begin{array}{cc}
J & 0 \\
0 & J
\end{array}\right), \quad \text { где } J=\left(\begin{array}{cccc}
0 & 0 & 1_{N} & 0 \\
0 & 1_{\infty} & 0 & \\
1_{N} & 0 & 0 & 0 \\
0 & 0 & 0 & 1_{\infty}
\end{array}\right)
$$

с достаточно большим $N$.

Итак, $L \backslash G / L$ - абелева полугруппа с инволюцией. Ее неприводимые представления одномерны. С другой стороны, неприводимость представления пары $(G, L)$ равносильна неприводимости соответствующего представления шлейфа $\mathfrak{T}(G, L)$, а у неприводимого представления упорядоченной категории представления всех полугрупп эндоморфизмов неприводимы (см. [17, лемма III.4.3]). Отсюда также вытекает следующее утверждение.

Предложение 3.3. Сферические функиии пары $(G, L)$ являются гомоморфизмами ${ }^{1)}$ полугруппы $L \backslash G / L$ в мультипликативную группу $\mathbb{C}^{\times}$.

3.4. Самоподобие. Рассмотрим чистую пару $(G, L)$. Рассмотрим ее подгруппу $G^{\alpha}$, как в разд. 1.8 .

Лемма 3.4. Образ $G^{\alpha}$ в $L^{\alpha} \backslash G / L^{\alpha}$ является центральной полугруппой, канонически изоморбной $L \backslash G / L$.

1) Разумеется, не произвольными. 
Доказательство. Изоморфизм индуцируется отображением «сдвига» $G \rightarrow$ $G^{\alpha}$, т. е. $g \mapsto\left(\begin{array}{ll}1 & 0 \\ 0 & g\end{array}\right)$. Пусть $g \in G, h \in G^{\alpha}$. Легко видеть, что при достаточно большом $m$

$$
g\left(\Theta_{m}^{[\alpha]} h \Theta_{m}^{[\alpha]}\right)=\left(\Theta_{m}^{[\alpha]} h \Theta_{m}^{[\alpha]}\right) g \Longrightarrow L^{\alpha} \cdot g \Theta_{m}^{[\alpha]} h \cdot L^{\alpha}=L^{\alpha} \cdot h \Theta_{m}^{[\alpha]} g \cdot L^{\alpha} .
$$

Далее заметим, что при $\alpha<\beta$ есть естественное отображение $K^{\beta} \backslash G / K^{\beta} \rightarrow$ $K^{\alpha} \backslash G / K^{\alpha}$, которое может быть задано также формулой

$$
\Pi: \mathfrak{g} \mapsto \mu_{\beta, \alpha} \circ \mathfrak{g} \circ \lambda_{\alpha, \beta} .
$$

Лемма 3.5. Проекиия П: $K^{\beta} \backslash G^{\beta} / K^{\beta} \rightarrow K^{\alpha} \backslash G^{\alpha} / K^{\alpha}$ чентралъных полугрупп совпадает с их изоморфизмом, индуиированным изоморфизмом сдвига $G^{\beta} \rightarrow G^{\alpha}$.

Доказательство. Элемент $g \in G^{\alpha}$ и соответствующий ему элемент $\widetilde{g} \in G^{\beta}$ сопряжены в $G^{\alpha}$ с помощью элемента группы $K^{\alpha}$.

Поэтому полугруппа $L \backslash G / L$ действует во всем пространстве $H$. Если представление $\rho$ неприводимо, то эта полугруппа действует скалярными операторами. Соответствующий характер совпадает со сферическими функциями групп $\left(G^{\alpha}, K^{\alpha}\right)$, действующих в $H$.

3.5. Самоограничение. Проведем сначала доказательство в случае $(G, L)=$ $(\mathrm{GL}(\infty, \mathbb{R}), \mathrm{O}(\infty))$. Мы сохраняем обозначения разд. 1.11.

Отображение $\tau: \mathbb{N} \rightarrow \Xi$ определяет 0-1-матрицу, обозначим ее через $A$, ее норма равна 1. Согласно теореме 1.1, представления тяжелых групп продолжаются по слабой непрерывности до представлений полугрупп сжатий. В частности, матрице $A$ соответствует оператор $\pi(A)$ в пространстве любого непрерывного представления $\pi$ группы $L$ в гильбертовом пространстве $H$.

Лемма 3.6. Оператор $\pi(A)$ - изометричное отображение пространства $H$ на подпространство $H^{[\Xi]}$.

Доказательство. Прежде всего, $(\pi(A))^{*} \pi(A)=\pi\left(A^{*} A\right)=\pi(1)=1$. Поэтому $\pi(A)$ - изометричное вложение. Аналогично, $\pi(A)(\pi(A))^{*}=\pi\left(A A^{*}\right)$. Оператор $A A^{*}$ - это проектор на $\ell_{2}(\Xi)$ в пространстве $\ell_{2}(\mathbb{N})$. Далее мы опять ссылаемся на явный вид представлений тяжелых групп [25].

Рассмотрим допустимое представление $\rho$ пары $(G, L)$. Обозначим через $\pi$ ограничение представления $\rho$ на $L$. Продолжим $\pi$ на полугруппу сжатий.

Лемма 3.7. Оператор $\pi(A)$ сплетает представление $\pi$ пары $(G, L)$ в пространстве $H$ и представление $\pi$ о $i_{\tau}$ в пространстве $H^{[\Xi]}$.

Доказательство. Рассмотрим последовательность матриц-перестановок $B_{m}$, сходящуюся слабо к $A$. Тогда $\pi\left(B_{m}\right)$ сходится слабо к $\pi(A)$. Группа $G$ является индуктивным пределом редуктивных групп $G(n)$. Для каждой группы $G(n)$ оператор $\pi\left(B_{m}\right)$ с достаточно большим номером $m$ является сплетающим. Поэтому предел является сплетающим оператором для всей группы $G$.

Пусть $L=L_{1} \times \cdots \times L_{p}$. Каждый из множителей - это группа унитарных операторов в некотором пространстве $\ell_{1}=\ell_{2}(\mathbb{N})$. Мы разбиваем каждую копию $\mathbb{N}$ на два счетных подмножества, $\mathbb{N}=\Xi_{j} \cup \Omega_{j}$. Фиксируем биекции $\tau_{j}: \mathbb{N} \rightarrow \Xi_{j}$. Далее повторяем все те же самые слова. 
3.6. Мантия. Теорема о мантии следует из теоремы мультипликативности и леммы о самоограничении.

3.7. Сферический характер, см. разд. 1.12. Рассмотрим $(G, L)$-пару, действующую в прямой сумме пространств $\ell_{2}$, как в разд. 2.1-2.2. Рассмотрим все базисные векторы $e_{\mu}$ в $\bigoplus \ell_{2}$, которые фиксируются группой $L$. Рассмотрим подгруппу $G_{\min } \subset G$, стабилизирующую все $e_{\mu}$.

Пример. Пусть $(G, L)=(\mathrm{GL}(7+2 \infty, \mathbb{R}), \mathrm{O}(\infty) \times \mathrm{O}(\infty))$. Тогда $G_{\min }=$ $\operatorname{GL}(2 \infty, \mathbb{R})$.

Предложение 3.8. Мантия пары $(G, L)$ содержсит иентральную полугруппу, канонически изоморфную $L \backslash G_{\min } / L$.

Объясним это на примере. Пусть $(G, L)=(\mathrm{U}(p+\infty) \times \mathrm{U}(\infty), \mathrm{U}(\infty))$, причем подгруппа $L \simeq \mathrm{U}(\infty)$ вложена в произведение по диагонали, и $G_{\min }=\mathrm{U}(\infty) \times$ $\mathrm{U}(\infty)$. Переобозначим $(G, L)$ как $(\mathrm{U}(p+2 \infty) \times \mathrm{U}(2 \infty), \mathrm{U}(2 \infty))$, будем записывать ее элементы как пары блочных унитарных матриц $(g, h)$, где $g$ имеет порядок $p+\infty+\infty$, а $h-$ порядок $\infty+\infty$. Рассмотрим подгруппу $Z \simeq \mathrm{U}(\infty) \times \mathrm{U}(\infty)$, состоящую из пар матриц

$$
\left\{\left(\begin{array}{lll}
1 & & \\
& 1 & \\
& & d_{1}
\end{array}\right),\left(\begin{array}{ll}
1 & \\
& d_{2}
\end{array}\right)\right\},
$$

где $d_{1}, d_{2} \in \mathrm{U}(\infty)$. В ней рассмотрим подгруппу $M$, состоящую из пар, удовлетворяющих условию $d_{1}=d_{2}$. Тогда мантия - это полугруппа $M \backslash G / M$. В нее вкладывается в качестве центральной полугруппы $M \backslash Z / M$. Центральность версия леммы 3.4 .

\section{§4. Обсуждение}

4.1. Общие гипотезы. Пусть пара $(G, L)$ конечна, см. разд. 2.2.

(a) Унитарные представления пары $(G, L)$ имеют тип $I$.

(b) Существует лишь счетное число неприводимых представлений с данным сферическим характером.

(c) Пусть $(G, L) \rightarrow\left(G^{\prime}, L^{\prime}\right)$ - вложение пар, причем вложение $L \rightarrow L^{\prime}$ правильное в смысле разд. 2.1. Тогда ограничение неприводимого представления $\rho$ пары $\left(G^{\prime}, L^{\prime}\right)$ на $(G, L)$ имеет чисто дискретный спектр. В частности, это так для тензорных произведений неприводимых представлений.

(d) Возможна явная классификация сферических представлений. Точнее, все они получаются конструкцией, описанной в [17, IX.5] (разница между симметрическими и несимметрическими $(G, L)$-парами объясняется в [17, IX.5.6]).

(е) В [20] для элемента $\mathfrak{g} \in L^{\alpha} \backslash G / L^{\beta}$ были построены спектральные данные: «характеристическая функция» (внутренняя функция матричного аргумента) и «собственная поверхность» (гиперповерхность в грассманиане). По этим данным $\mathfrak{g}$ однозначно восстанавливается.

4.2. Характеристические функции: пример. Мы рассмотрим следующую $(G, K)$-пару (возможно, не самую естественную с точки зрения теории представлений, но простейшую с точки зрения характеристических функций). Рассмотрим группу $H=\mathrm{U}(\alpha+n \infty)$ и ее подгруппу $K=\mathrm{U}(\infty)$, состоящую из 
матриц вида

$$
\left(\begin{array}{cccc}
1_{\alpha} & 0 & \ldots & 0 \\
0 & u & \ldots & 0 \\
\vdots & \vdots & \ddots & \vdots \\
0 & 0 & \ldots & u
\end{array}\right)
$$

где $u \in \mathrm{U}(\infty)$. Положим $G=H \times K$, подгруппа $K \subset G$ вложена в произведение по диагонали. Тогда двойные классы смежности $K \backslash G / K$ находятся во взаимно однозначном соответствии с классами сопряженности $\mathrm{U}(\alpha+n \infty)$ относительно подгруппы $K$.

Запишем элемент $g \in \mathrm{U}(\alpha+n \infty)$ как блочную матрицу

$$
g=\left(\begin{array}{cccc}
a & b_{1} & \ldots & b_{n} \\
c_{1} & d_{11} & \ldots & d_{1 n} \\
\vdots & \vdots & \ddots & \vdots \\
c_{p} & d_{n 1} & \ldots & d_{n n}
\end{array}\right)
$$

Пусть $S$ - матрица размера $p \times p$. Пусть $x_{1}, \ldots, x_{p}-$ векторы из $\ell_{2}$, и пусть $p, q \in \mathbb{C}^{\alpha}$. Рассмотрим следующий «извращенный аналог» характеристического уравнения $A y=\lambda y$ :

$$
\left(\begin{array}{c}
q \\
x_{1} \\
\vdots \\
x_{n}
\end{array}\right)=\left(\begin{array}{cccc}
a & b_{1} & \ldots & b_{n} \\
c_{1} & d_{11} & \ldots & d_{1 n} \\
\vdots & \vdots & \ddots & \vdots \\
c_{p} & d_{n 1} & \ldots & d_{n n}
\end{array}\right)\left(\begin{array}{c}
q \\
s_{11} x_{1}+\cdots+s_{1 n} x_{n} \\
\vdots \\
s_{n 1} x_{1}+\cdots+s_{n n} x_{n}
\end{array}\right)
$$

Исключая переменные $x_{1}, \ldots, x_{n}$, мы получаем зависимость

$$
q=\chi_{g}(S) p,
$$

где $\chi_{g}(S)$ - матрица размера $\alpha \times \alpha$. Эту рациональную функцию $\operatorname{Mat}(n) \rightarrow$ $\operatorname{Mat}(\alpha)$ мы назовем характеристической функиией.

Собственную поверхность $\Xi_{g} \subset \operatorname{Mat}(n)$ мы определим уравнением

$$
\operatorname{det}\left(\left(\begin{array}{ccc}
d_{11} & \ldots & d_{1 n} \\
\vdots & \ddots & \vdots \\
d_{n 1} & \ldots & d_{n n}
\end{array}\right)-\left(\begin{array}{ccc}
s_{11} & \ldots & s_{1 n} \\
\vdots & \ddots & \vdots \\
s_{n 1} & \ldots & s_{n n}
\end{array}\right)\right)=0 .
$$

Мы рассматриваем ее как дивизор в смысле алгебраической геометрии, т. е. набор неприводимых компонент алгебраического многообразия с учетом кратностей.

Теорема 4.1. (а) Характеристическая функиия и собственная поверхность зависят лищь от двойного класса смежнноти $\mathfrak{g} \in K \backslash G / K$, содержсащего $g$.

(b) $\chi_{\mathfrak{g}}(S)$ есть рациональная функиия от $S$, причем ее особенности содержатся в $\Xi_{\mathfrak{g}}$.

(c) Выполнено следующее тождество:

$$
\chi_{\mathfrak{g}_{1}}(S) \chi_{\mathfrak{g}_{2}}(S)=\chi_{\mathfrak{g}_{1} \circ \mathfrak{g}_{2}}(S) .
$$

Обозначим через $\mathrm{B}_{k}$ множество матриц размера $k \times k$ с нормой $<1$. 
Теорема 4.2. (а) Отображение $\chi_{\mathfrak{g}}$ голоморфно на $\mathrm{B}_{n}$ и переводит $\mathrm{B}_{n}$ в $\mathrm{B}_{\alpha}$.

(b) Если $\chi_{\mathfrak{g}}$ определено в точке $S \in \mathrm{U}(n)$, то $\chi_{\mathfrak{g}}(S) \in \mathrm{U}(\alpha)$.

4.3. Внутренние отображения. Назовем рациональное отображение $\operatorname{Mat}(n) \rightarrow \operatorname{Mat}(\alpha)$ внутренним, если оно удовлетворяет заключению предыдущей теоремы.

Напомним, что внутренние отображения единичного круга $\mathrm{B}_{1}$ в себя - классический предмет теории функций (см., например, [4]), а внутренние отображения $\mathrm{B}_{1} \rightarrow \mathrm{B}_{n}-$ классический предмет теории операторов, см. [33].

Обозначим через $\operatorname{Int}\left(\mathrm{B}_{n}, \mathrm{~B}_{\alpha}\right)$ множество всех внутренних отображений $\mathrm{B}_{n} \rightarrow$ $\mathrm{B}_{\alpha}$, а через $\operatorname{Int}^{\circ}\left(\mathrm{B}_{n}, \mathrm{~B}_{\alpha}\right)$ множество характеристических функций $\chi_{\mathfrak{g}}$.

Теорема 4.3. (a) Ecлu $F_{1}, F_{2} \in \operatorname{Int}^{\circ}\left(\mathrm{B}_{n}, \mathrm{~B}_{\alpha}\right)$, mo $F_{1} F_{2} \in \operatorname{Int}^{\circ}\left(\mathrm{B}_{n}, \mathrm{~B}_{\alpha}\right)$.

(b) Ecлu $F_{1} \in \operatorname{Int}^{\circ}\left(\mathrm{B}_{n}, \mathrm{~B}_{\alpha}\right), F_{2} \in \operatorname{Int}^{\circ}\left(\mathrm{B}_{n}, \mathrm{~B}_{\beta}\right), m o F_{1} \oplus F_{2} \in \operatorname{Int}^{\circ}\left(\mathrm{B}_{n}, \mathrm{~B}_{\alpha+\beta}\right)$.

(c) Ecлu $F_{1} \in \operatorname{Int}^{\circ}\left(\mathrm{B}_{n}, \mathrm{~B}_{\alpha}\right), F_{2} \in \operatorname{Int}^{\circ}\left(\mathrm{B}_{n}, \mathrm{~B}_{\beta}\right), m o F_{1} \otimes F_{2} \in \operatorname{Int}^{\circ}\left(\mathrm{B}_{n}, \mathrm{~B}_{\alpha \beta}\right)$.

(d) Ecли $F_{1} \in \operatorname{Int}^{\circ}\left(\mathrm{B}_{n}, \mathrm{~B}_{m}\right), F_{2} \in \operatorname{Int}^{\circ}\left(\mathrm{B}_{m}, \mathrm{~B}_{k}\right), m o F_{2} \circ F_{1} \in \operatorname{Int}^{\circ}\left(\mathrm{B}_{n}, \mathrm{~B}_{k}\right)$.

(e) $E c л u F_{1} \in \operatorname{Int}\left(\mathrm{B}_{n}, \mathrm{~B}_{\alpha}\right), F_{2} \in \operatorname{Int}\left(\mathrm{B}_{n}, \mathrm{~B}_{\beta}\right), F_{1} \oplus F_{2} \in \operatorname{Int}^{\circ}\left(\mathrm{B}_{n}, \mathrm{~B}_{\alpha+\beta}\right), m o$ $F_{1} \in \operatorname{Int}^{\circ}\left(\mathrm{B}_{n}, \mathrm{~B}_{\alpha}\right), F_{2} \in \operatorname{Int}^{\circ}\left(\mathrm{B}_{n}, \mathrm{~B}_{\beta}\right)$.

Гипотеза 4.4. $\operatorname{Int}^{\circ}\left(\mathrm{B}_{n}, \mathrm{~B}_{\alpha}\right)=\operatorname{Int}\left(\mathrm{B}_{n}, \mathrm{~B}_{\alpha}\right)$.

4.4. Почему мы рассматриваем лишь такие пары? Есть много вложений группы $\mathrm{U}(n)$ в унитарные группы большого размера $\mathrm{U}(N)$. Например, $g \mapsto g \otimes g$ задает вложение $\mathrm{U}(n) \rightarrow \mathrm{U}\left(n^{2}\right)$. Однако для $g \in \mathrm{U}(\infty)$ матрица $g \otimes g$ не является финитной. То есть формально вложения $\mathrm{U}(n)$ в $\mathrm{U}(\cdot)$, отличные от прямых вложений, не выживают в пределе при $n \rightarrow \infty$.

Понятно, что это довод не достаточен. Можно искать естественные подгруппы в полной операторной группе в $\ell_{2}(\mathbb{N} \times \mathbb{N})$, содержащие матрицы $g \otimes g$. Но таких подгрупп не видно ${ }^{1)}$.

4.5. Сферичность. Есть примеры сферических пар, не являющихся чистыми, скажем $(\mathrm{U}(1+\infty) \times \mathrm{U}(\infty), \mathrm{U}(\infty)),(\mathrm{O}(1+2 \infty), \mathrm{U}(\infty))$, см. [30].

Кроме того, есть много случаев сферичности относительно подгрупп вида $L \times$ $M$, где $L$ - тяжелая подгруппа, а $M$ - компактная (например, упоминавшаяся выше пара $(\mathrm{U}(p+\infty), \mathrm{U}(p) \times \mathrm{U}(\infty))$.

4.6. Симметрические группы. Похожая картина возникает и для симметрических групп, пример обсуждается в [19].

\section{ЛитеРАТУРА}

[1] A. Borodin, G. Olshanski, Harmonic analysis on the infinite-dimensional unitary group and determinantal point processes, Ann. of Math. (2), 161:3 (2005), 1319-1422.

[2] M. Brion, Classification des espaces homogènes sphériques, Compositio Math., 63:2 (1987), 189-208.

[3] М. С. Бродский, Унитарные операторные узлы и их характеристические функиии, УМН, 33:4(202) (1978), 141-168.

[4] Дж. Гарнетт, Ограниченные аналитические функиии, Мир, М., 1984.

[5] И. М. Гельфанд, Сферические функиии на римановых симметрических пространстваx, ДАН СССР, 70 (1950), 5-8.

[6] Ж. Диксмье, $C^{*}$-алгебры и их представления, Наука, М., 1974.

1) Например, подгруппа порожденная финитными унитарными матрицами и матрицами $g \otimes g$, разлагается в прямое произведение двух порождающих подгрупп. 
[7] С. Хелгасон, Дифберенциальная геометрия, группь Ли и симметрические пространства, Факториал Пресс, М., 2005.

[8] Р. С. Исмагилов, Элементарные сферические функиии на группе $S L(2, P)$ над полем $P$, не являющимся локально компактным, относительно подгруппы матрии с иелыми элементами, Изв. АН СССР, сер. матем., 31:2 (1967), 361-390.

[9] Р. С. Исмагилов, Сферические функции над нормированным полем, поле вычетов которого бесконечно, Функц. анализ и его прил., 4:1 (1970), 42-51.

[10] F. Knop, Semisymmetric polynomials and the invariant theory of matrix vector pairs, Represent. Theory, 5 (2001), 224-266.

[11] T. H. Koornwinder, Jacobi functions and analysis on noncompact semisimple Lie groups, in: Special Functions: Group Theoretical Aspects and Applications, Math. Appl., Reidel, Dordrecht, 1984, 1-85.

[12] M. Krämer, Sphärische Untergruppen in kompakten zusammenheängenden Liegruppen, Compositio Math., 38:2 (1979), 129-153.

[13] М. С. Лившиц, Об одном классе линейных операторов в гилъбертовом пространстве, Матем. сб., 19(61):2 (1946), 239-262.

[14] I. G. Macdonald, Symmetric Functions and Hall Polynomials, 2nd ed. With contributions by A. Zelevinsky, Oxford Univ. Press, New York, 1995 [Имеется перевод первого издания: И. Макдональд, Симметрические функиии и многочлень Холла, Мир, М., 1984.].

[15] И. В. Микитюк, Об интегрируемости инвариантных гамильтоновых систем с однородным конфигурационным пространством, Матем. сб., 129:4 (1986), 514534.

[16] Ю. А. Неретин, Категории бистохастических мер и представления некоторьх бесконечномерных групп, Матем. сб., 183:2 (1992), 52-76.

[17] Ю. А. Неретин, Категории симметрий и бесконечномерные группь, Эдиториал УPCC, M., 1998, 2009.

[18] Yu. A. Neretin, Hua-type integrals over unitary groups and over projective limits of unitary groups, Duke Math. J., 114:2 (2002), 239-266.

[19] Yu. A. Neretin, Infinite tri-symmetric group, multiplication of double cosets, and checker topological field theories, Int. Math. Res. Notices, 2011, doi: 10.1093/imrn/ rnr027; http://arxiv.org/abs/0909.4739.

[20] $\mathrm{Yu}$. A. Neretin, Multi-operator colligations and multivariate characteristic functions, Analysis and Mathematical Physics, в печати; http://arxiv.org/abs/1006.2275.

[21] Н. И. Нессонов, Фактор-представления группы $\mathrm{GL}(\infty)$ и допустимые представления GL $(\infty)^{X}$, Матем. физика, анализ, геометрия, 10:4 (2003), 167-187.

[22] Н. И. Нессонов, Фактор-представления группъ $\mathrm{GL}(\infty)$ и допустимые представления GL $(\infty)^{X}$. II, Матем. физика, анализ, геометрия, 10:4 (2003), 524-556.

[23] Г. И. Ольшанский, Новые «болъшие» группы типа I, в кн.: Итоги науки и техники. Современные проблемы математики, т. 16, ВИНИТИ, М., 1980, 31-52.

[24] Г. И. Ольшанский, Унитарные представления бесконечномерных пар $(G, K) u$ формализм Хау, ДАН СССР, 269:1 (1983), 33-36.

[25] Г. И. Ольшанский, Бесконечномерные классические группы конечного $R$-ранга: описание представлений и асимптотическая теория, Функц. анализ и его прил., 18:1 (1984), 28-42.

[26] Г. И. Ольшанский, Унитарные представления группь $\mathrm{SO}_{0}(\infty, \infty)$ как предель унитарных представлений групn $\mathrm{SO}_{0}(n, \infty)$ рри $n \rightarrow \infty$, Функц. анализ и его прил., 20:4 (1986), 46-57.

[27] Г. И. Ольшанский, Метод голоморфных расширений в теории унитарных представлений бесконечномерных классических групп, Функц. анализ и его прил., 22:4 (1988), 23-37. 
[28] G. I. Olshanski, Unitary representations of infinite dimensional pairs $(G, K)$ and the formalism of R. Howe, in: Representation of Lie Groups and Related Topics, Adv. Stud. Contemp. Math., vol. 7, Gordon and Breach, New York, 1990, 269-463.

[29] Г. И. Ольшанский, Унитарные представления $(G, K)$-пар, связанных с бесконечной симметрической группой $S(\infty)$, Алгебра и анализ, 1:4 (1989), 178-209.

[30] G. I. Olshanski, Caractères généralisés du groupe $U(\infty)$ et fonctions intèrieures, C. R. Acad. Sci. Paris. Sér. 1, 313:1 (1991), 9-12.

[31] G. I. Olshanski, The problem of harmonic analysis on the infinite-dimensional unitary group, J. Funct. Anal., 205:2 (2003), 464-524.

[32] D. Pickrell, Separable representations for automorphism groups of infinite symmetric spaces, J. Funct. Anal., 90:1 (1990), 1-26.

[33] В. П. Потапов, Мультипликативная структура $J$-нерастягивающей матричной функиии, Труды ММO, 4 (1955), 125-236.

[34] S. Strătilă, D. Voiculescu, Representations of AF-Algebras and of the Group $U(\infty)$, Lecture Notes in Math., vol. 486, Springer-Verlag, New York-Heidelberg-Paris, 1975.

[35] А. М. Вершик, С. В. Керов, Характеры и фактор-представления бесконечномерной унитарной группъ, ДАН СССР, 267:2 (1982), 272-276.

University of Vienna

Институт теоретической и экспериментальной физики

Поступило в редакцию

Московский государственный университет

e-mail: neretin@mccme.ru

Заведующая редакцией и научный редактор Г. М. Цукерман

Сдано в набор 24.06.2011. Подписано к печати 22.7.2011. Формат 70×100/16

Печать офсетная. Усл. печ. л. 7,8. Усл. кр.-отт. 1,8 тыс. Бум. л. 3,0

Уч.-изд. л. 8,0. Тираж 215 экз. Заказ 1506.

Учредитель: Российская академия наук

Адрес редакции: 117966 Москва, ГСП-1, ул. Губкина 8, комн. 624. Тел. 938-37-56

Издатель: Российская академия наук, Издательство «Наука»,

117997 Москва, Профсоюзная, ул. 90

Отпечатано в ППП «Типография «Наука», 121099 Москва, Шубинский пер., 6 